J. Clin. Chem. Clin. Biochem.

Vol. 26, 1988, pp. $75-78$

(C) 1988 Walter de Gruyter \& Co.

Berlin - New York

\title{
Ein einfacher und schneller Enzymimmunoassay zur quantitativen Bestimmung des C-reaktiven Proteins
}

\author{
Von W. Schößler
}

Staatliches Institut für Immunpräparate und Nährmedien, Berlin, DDR

G. Töpfer

Institut für Klinische Chemie und Laboratoriumsdiagnostik, Bezirkskrankenhaus Görlitz, DDR und

H.-J. Rüger

Staatliches Institut für Immunpräparate und Nährmedien, Berlin, DDR

(Eingegangen am 14. Mai/21. Dezember 1987)

Zusammenfassung: Vorliegende Arbeit beschreibt einen einfachen und schnellen Enzymimmunoassay zur quantitativen Bestimmung des C-reaktiven Proteins (CRP). Hierbei konkurriert das im Serum befindliche CRP mit einem enzymmarkierten CRP (Enzym: alkalische Phosphatase) um die an der festen Phase befindlichen Antikörper (kompetitiver Enzymimmunoassay). Die Nachweisgrenze wurde mit $10 \mu \mathrm{g} / 1 \mathrm{ermittelt.} \mathrm{Die}$ Präzision in der Serie im Grenzbereich „normal-pathologisch“ (5 mg/l) wurde mit 2,9\%, die Präzision von Tag zu Tag bei $5 \mathrm{mg} / 1 \mathrm{mit}$ 4,5\% ermittelt. Der Zeitbedarf für die Ausführung des gesamten Tests beträgt etwa 2 Stunden.

\section{A simple and fast enzyme immunoassay for the quantitative determination of $C$-reactive protein}

Summary: A simple and rapid enzyme immunoassay for the quantitative determination of C-reactive protein (CRP) is described.

The principle is based on the competitive binding of the enzyme-labelled CRP (enzyme: alkaline phosphatase) and the serum CRP to solid phase-bound antibodies. The detection limit was $10 \mu \mathrm{g} / \mathrm{l}$. The precision in the series in the border-line "normal" to "pathological" $(5 \mathrm{mg} / \mathrm{l})$ was $2.9 \%$; the precision from day to day at the concentration of $5 \mathrm{mg} / \mathrm{l}$ was $4.5 \%$.

Only $2 \mathrm{~h}$ are required for the whole test.

\section{Einftihrung}

Die Bestimmung des C-reaktiven Proteins (CRP) als diagnostischer Marker eines entzündlichen Prozesses, z. B. bei Infektionen, Tumorerkrankungen, Verbrennungen, nach chirurgischen Eingriffen, aber auch als Indikator eines Myokardinfarktes oder einer Rejektionskrise nach Transplantationen erlangte in den letzten Jahren zunehmende Bedeutung, so daß das CRP als zuverlässigstes Akute-Phase-Protein angesehen werden muß $(1,2)$. Eine besondere Bedeutung hat die Bestimmung des CRP bei neonatalen Infektionen $(1,2)$.

Die diagnostische Relevanz des C-reaktiven Proteins ist vor allem auf seine Dynamik hinsichtlich der Geschwindigkeit und Größe der Konzentrationsänderung zurückzuführen. So setzt der Konzentrations- 
anstieg des CRP bereits wenige Stunden (6h) nach Beginn des zugrunde liegenden entzündlichen Prozesses ein. Die biologische Halbwertzeit des CRP beträgt etwa einen Tag. Während die Referenzwerte bei gesunden Erwachsenen sowie Neugeborenen etwa $1 \mathrm{mg} / \mathrm{l}$ betragen, können die CRP-Konzentrationen bei entzündlichen Prozessen bis auf das 1000-fache ansteigen.

Obwohl in den letzten Jahren eine Reihe von immunchemischen Methoden zur quantitativen Bestimmung des CRP wie die einfache radiale Immundiffusion sowie die Nephelometrie und Turbidimetrie beschrieben wurden, stehen einer breiten Nutzung dieser Methoden in der Praxis eine Reihe von experimentellen und apparativen Problemen im Wege. Die in den letzten Jahren beschriebenen Radio- $(3,4)$, Fluoro(5) und Enzymimmunoassays (6-8) zur Bestimmung des CRP entsprachen hinsichtlich ihrer Praktikabilität nicht immer der dominierenden Bedeutung des CRP als Akute-Phase-Protein, da die für die Durchführung des Testes erforderlichen Zeiten zu lang waren. Eine Akute-Phase-Diagnostik erfordert eine zuverlässige, schnelle und hinreichend genaue Bestimmungsmethode.

In der vorliegenden Arbeit wird ein einfacher und schneller Enzymimmunoassay (EIA) zur quantitativen Bestimmung des CRP beschrieben.

\section{Material und Methoden}

Abweichend von den bisher beschriebenen Enzymimmunoassays zum Nachweis des CRP wählten wir das Prinzip eines kompetitiven Assays (Abb. 1). Hierbei konkurriert das im Serum befindliche CRP mit einem enzymmarkierten CRP um die an der festen Phase befindlichen Antikörper. Nach Ablauf der Immunreaktion wird die Enzymaktivität, die der Konzentration des im Serum befindlichen CRP reziprok proportional ist, durch Umsetzung des für das Enzym charakteristischen Substrates und photometrische Messung des Reaktionsproduktes ermittelt.

\section{Isolierung von CRP}

C-reaktives Protein wurde durch Affinitätschromatographie an mit Antikörpern beladener Sepharose isoliert. Hierzu wurde aus einem Kaninchen-anti-human CRP-Antiserum (s. u.) das Kaninchen-IgG durch mehrfache Fällung mit Ammoniumsulfat (40\%ige Sättigung) und nachfolgende Ionenaustauschchromatographie an DEAE-Sephadex A 50 (Pharmacia, Schweden) mit einem $0,02 \mathrm{~mol} / \mathrm{l}$ und $0,03 \mathrm{~mol} / \mathrm{l}$ Citronensäure-PhosphatPuffer (Mc Ilvain-Puffer), pH 7,4, isoliert und anschließend an CNBr-aktivierte Sepharose (Pharmacia, Schweden) entsprechend den Angaben des Herstellers gebunden.

Die so beladene Matrix wurde zur Isolierung des CRP aus einem Serumpool erhöhter CRP-Konzentrationen eingesetzt. Nach Elution der ungebundenen Proteine wurde das CRP mit $1 \mathrm{~mol} / \mathrm{l} \mathrm{KSCN}$ in phosphat-gepufferter isotoner $\mathrm{NaCl}$-Lösung,

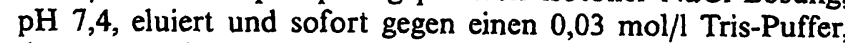
der $0,3 \mathrm{~mol} / 1 \mathrm{NaCl}$ enthielt, $\mathrm{pH} 8,0$, dialysiert. Die Reinheitsprüfung erfolgte mit der SDS-Polyacrylamidelektrophorese. In
Bindung des CRP-Antikörpers an Polystyren

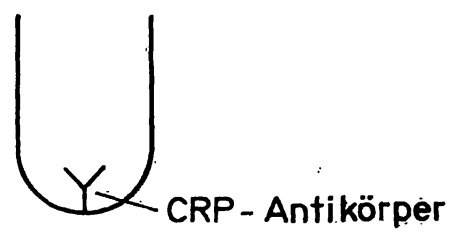

Kompetitive Bindung des CRP und des

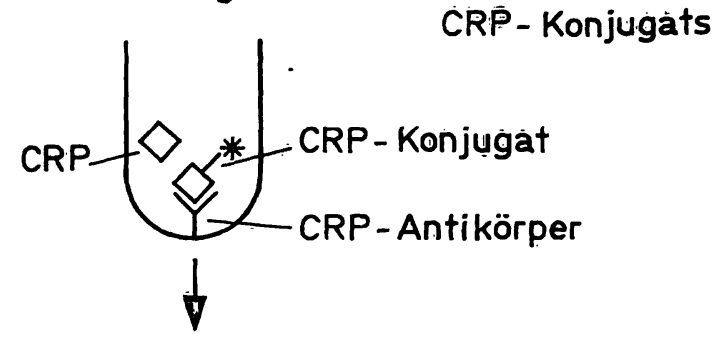

Bestimmung der Enzymaktivität

Fig. 1. Darstellung des Prinzips des Enzymimmunoassays zur Bestimmung des C-reaktiven Proteins (CRP).

einigen Fällen erwies sich die Entfernung von Begleitproteinen durch Immunadsorption mit an Sepharose gebundenen Immunglobulinen gegen humane Serumproteine als erforderlich. Nach Konzentrierung wurde das CRP portioniert und bei $-20^{\circ} \mathrm{C}$ eingefroren.

Die Ausbeuten lagen zwischen $80-90 \%$.

\section{Gewinnung des Antiserums}

Die Immunisierung von Kaninchen mit CR̈P erfolgte durch subkutane Injektion von je $1 \mathrm{mg}$ Protein in komplettem Freund'schen Adjuvans in die Fußballen. Die nachfolgenden Immunisierungen erfolgen mit je $500 \mu \mathrm{g}$ CRP in inkomplettem Freundschen Adjuvans. Das so erhaltene Antiserum wurde mittels glutaraldehydvernetztem Normalserum adsorbiert. Nunmehr erwies sich das Antiserum in der Immunelektrophorese als monospezifisch gegen humanes CRP.

\section{Isolierung der Antikörper}

Die spezifischen - gegen humanes CRP gerichteten - Antikörper wurden mittels Affinitätschromatographie aus dem entsprechenden Antiserum isoliert. Hierzu wurde humanes CRP an $\mathrm{CNBr}$-aktivierte Sepharose (Pharmacia, Schweden) entsprechend den Angaben des Herstellers gebunden und nachfolgend mit der mittels Ammoniumsulfat präzipitierten Immunglobulinfraktion des Antiserums beladen. Nach Elution der ungebundenen Proteine mit phosphat-gepufferter isotoner $\mathrm{NaCl}$ Lösung, $\mathrm{pH} 7,4$, wurden die spezifischen Antikörper mit $\mathrm{HCl}$ Glycin-Puffer, pH 2,8, eluiert und anschließend sofort neutralisiert und gegen phosphat-gepufferte isotone $\mathrm{NaCl}$-Lösung $\mathrm{pH}$ 7,4 , die $0,2 \mathrm{~g} / 1$ Natriumazid enthielt, dialysiert. Die Reinheit der gewonnenen Antikörper wurde mittels Immunelektrophorese und Polyacrylamid-Disk-Elektrophorese getestet.

\section{Herstellung des Konjugatès}

Die Kopplung des Enzyms alkalische Phosphatase (EC 3.1.3.1) an das CRP erfolgte im wesentlichen nach der Methode von Engvall \& Perlmann (9). Beispielsweise wurden $1 \mathrm{mg}$ alkalische Phosphatase (1500 U/mg; Forschungsinstịtut für Medizinische 
Diagnostik Dresden) mit $390 \mu \mathrm{g}$ CRP mit Glutaraldehyd (Serva) in einer Endkonzentration von $2 \mathrm{~g} / 1$ zur Reaktion gebracht, zwei Stunden bei Raumtemperatur inkubiert und anschließend gegen phosphat-gepufferte isotone $\mathrm{NaCl}$-Lösung und $0,03 \mathrm{~mol} / \mathrm{l}$ Tris-Puffer, $\mathrm{pH} 8,0$, der $1 \mathrm{mmol} / \mathrm{l} \mathrm{MgCl}_{2}$ und $0,2 \mathrm{~g} / 1 \mathrm{NaN}_{3}$ enthielt, dialysiert und schließlich mit $20 \mathrm{~g} / \mathrm{l}$ Humanserumalbumin ( $98 \%$, Serva) versetzt, portioniert und bei $-20^{\circ} \mathrm{C}$ gelagert.

\section{Durchführung des EIA}

Zur Ausführung des EIA wird im ersten Schritt der affinitätschromatographisch gereinigte Antikörper in der üblichen Art und Weise in 0,1 mol/1 Carbonat-Bicarbonat-Puffer, pH 9,6, an Polystyren-Röhrchen oder Mikrotestplatten (VEB Polyplast Halberstadt, DDR) oder aber kovalent nach vorheriger chemischer Modifizierung (10) des Polystyrens gebunden. Anschließend werden die überschüssigen Antikörpermoleküle durch Waschen mit phosphat-gepufferter isotoner $\mathrm{NaCl}$-Lösung, $\mathrm{pH} 7,4$, die $0,5 \mathrm{~g} / 1$ Tween 20 (Serva) und $0,2 \mathrm{~g} / 1 \mathrm{NaN}_{3}$ enthält, entfernt. Die so beladenen Träger können bei $-20^{\circ} \mathrm{C}$ gelagert werden, so daß sie bei Bedarf sofort zur Verfügung stehen. Das zu bestimmende Serum wird mit dem enzymmarkierten CRP in einer geeigneten Verdünnung $(1: 500)$ in phosphat-gepufferter isotoner $\mathrm{NaCl}$-Lösung, $\mathrm{pH} 7,4$, die $0,5 \mathrm{~g} / 1$ Tween $20,0,2 \mathrm{~g} / 1 \mathrm{NaN}_{3}$ und $10 \mathrm{~g} / 1$ Rinderserumalbumin $(98 \%$, Serva) enthält, gemischt und eine Stunde bei $45^{\circ} \mathrm{C}$ in den zuvor mit Antikörpern beladenen Polystyren-Trägern inkubiert. Um zu gewährleisten, daß die Absorption des zu bestimmenden Serums in den linearen Bereich der Standardkurve fällt, werden unbekannte Seren in der Regel in den Verdünnungen $1: 100$, und $1: 1000$ bestimmt. Nach Entfernung der nicht gebundenen Reaktanden durch Waschen mit o.g. Waschpuffer wird die Enzymaktivität durch Umsetzung von 4-Nitrophenylphosphat in $1 \mathrm{mmol} / 1$ Diethanolamin-Puffer, $\mathrm{pH} \mathrm{9,8}$, und photometrischer Messung des Reaktionsproduktes bei $405 \mathrm{~nm}$ nach Stoppen mit $2 \mathrm{~mol} / 1 \mathrm{NaOH}$ (11) bestimmt.

\section{Immunchemische Bestimmung des CRP}

Als Referenzmethoden wurden die einfache radiale Immundiffusion nach Mancini (Partigen-Platten, Behring-Werke) sowie die Laurell-rocket-Technik (12) eingesetzt.

\section{Ergebnisse}

Aus dem von uns gewählten Prinzip eines kompetitiven EIA resultiert eine reziproke Abhängigkeit der zu bestimmenden CRP $\div$ Konzentration von der Enzymaktivität. Eine charakteristische Standardkurve ist in Abbildung 2 dargestellt.

Die Nachweisgrenze des EIA, die sich aus der dreifachen Standardabweichung des Blindwertes ergibt, wurde mit einem CRP-freien Serum bestimmt. Sie wurde mit etwa $10 \mu \mathrm{g} / 1$ ermittelt. Der Meßbereich unseres EIA erstreckt sich bei einer 1:100 Serumverdünnung von $2-20 \mathrm{mg} / 1$ und bei einer $1: 1000 \mathrm{Se}-$ rumverdünnung von $20-200 \mathrm{mg} / \mathrm{l}$ (annähernd linearer Bereich der Standardkurve).

Die Präzision in der Serie $(n=20)$ (intra-assayVariationskoeffizient) wurde bei einer Konzentration von $2 \mathrm{mg} / \mathrm{l} \mathrm{mit} 12 \%$, bei $5 \mathrm{mg} / \mathrm{l} \mathrm{mit} 2,9 \%$ und bei 400

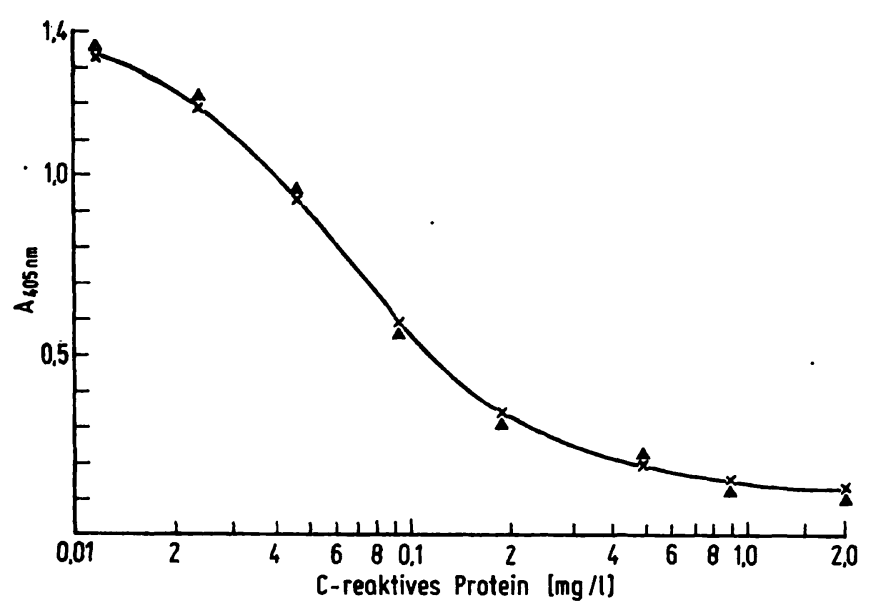

Fig. 2. Standardkurve des CRP im Enzymimmunoassay $\Delta$ gereinigtes CRP, $\times$ Behring CRP-Standard

$\mathrm{mg} / \mathrm{l} \mathrm{mit} 8 \%$ ermittelt; die Präzision von Tag zu Tag (inter-assay-Variationskoeffizient) wurde bei einer Konzentration von $2 \mathrm{mg} / \mathrm{l} \mathrm{mit} 15 \%$, bei $5 \mathrm{mg} / \mathrm{l} \mathrm{mit}$ $4,5 \%$ und bei $350 \mathrm{mg} / 1 \mathrm{mit} 10,5 \%$ bestimmt $(\mathrm{n}=20)$.

Die Richtigkeit des Enzymimmunoassays konnte durch Vergleich mit der einfachen radialen Immundiffusion (Mancini-Technik) und der Elektroimmunodiffusion (Laurell-rocket-Technik) bestätigt werden. Abbildung 3 zeigt die Regressionsgerade, berechnet nach der Methode der standardisierten Hauptkomponente, des Vergleiches des EIA mit der Elektroimmunodiffusion (EID) für 95 untersuchte Seren sowie die hieraus errechneten Korrelationskoeffizienten.

Die Spezifität unseres Testes wird im wesentlichen durch den an der festen Phase befindlichen Antikörper determiniert.

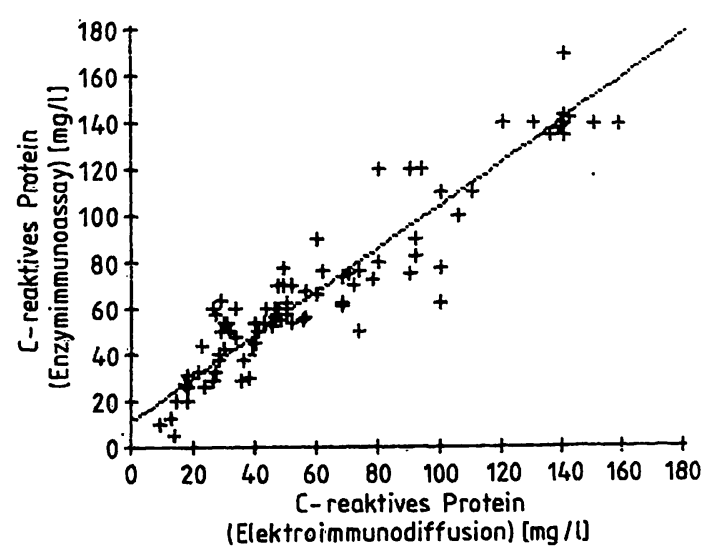

Fig. 3. Darstellung des Methodenvergleiches zwischen dem Enzymimmunoassay (EIA) und der Elektroimmunodiffusion (EID) durch Ermittlung der Regressionsgeraden sowie des Korrelationskoeffizienten ( $r$ ) für 95 untersuchte Seren (n). EIA $=0,94 \times$ EID + 11,2; $r=0,95$ 
Aus dem direkten Vergleich zwischen einem gereinigten CRP-Standard und einem kommerziellen CRP-Standard-Serum (Behring-Werke) läßt sich eine deutliche Interferenz nicht ableiten.

Der Zeitbedarf für die Ausführung des gesamten EIA beträgt etwa zwei Stunden und dürfte somit vielen Anforderungen der klinischen Praxis entsprechen. Hinzu kommt, daß für die Durchführung des Assays lediglich $10 \mu \mathrm{l}$ Serum benötigt werden.

\section{Diskussion}

Eine Akute-Phase-Diagnostik erfordert eine schnelle und kostengünstige Methode mit hinreichender Präzision insbesondere im Bereich „normal“ bis „pathologisch“. Der von uns vorgestellte Enzymimmunoassay gestattet die quantitative Bestimmung des C-reaktiven Proteins in etwa zwei Stunden und kommt somit den Anforderungen der klinischen Praxis weitgehend entgegen. Der Methodenvergleich mit der einfachen radialen Immundiffusion sowie mit der Elektroimmunodiffusion zeigt, daß die Richtigkeit unseres EIA gegeben ist, wenngleich die Regressionsgerade, berechnet nach der standardisierten Hauptkomponente (Abb. 3), die Ordinate bei $11,2 \mathrm{mg} / 1$ schneidet und somit auf eine Abweichung zu höheren Werten, insbesondere bei geringen Konzentrationen, hinweist. Dịe ermittelten Präzisionen entsprechen den Anforderungen, die an einen solchen Test zu stellen sind.

Auf Grund der vorliegenden Ergebnisse und Erfahrungen empfiehlt sich dieser Test zur einfachen und schnellen Bestimmung des CRP in der klinischen Routine. Gegenwärtig bemühen wir uns, den Zeitbedarf für die Durchführung des EIA weiter zu reduzieren und den Test für „bed-side“ Bedingungen nutzbar zu machen.

\section{Literatur}

1. Whicher, J. T. \& Dieppe, P. A. (1985) Clinics in Immunology and Allergy 5, 425-446.

2. Groth, J. \& Kaden, J. (1985) Z. Klin. Med. 40, 15571562.

3. Claus, D. R., Osmand, A. P. \& Gewurz, H. (1976) J. Lab. Clin. Med. 87, 120-128.

4. Shine, B., de Beer, F. C. \& Pepys, M. B. (1981) Clin. Chim. Acta $117,13-23$.

5. Nargessi, R. D., Shine, B. \& Landon, J. (1984) J. Immunol. Methods 71, 17-24.

6. Highton, J. \& Hession, P. (1984) J. Immunol. Methods 68, $185-192$.

7. Laurent, P., Marchand, B. \& Bienvenu, J. (1985) Clin. Biochem. 18, 272-275.

8. Bürger, W., Schmechta, H., Tambor, A., Porstmann, T. \& von Baehr, R. (1986) Z. Klin. Med. 41, 385-387.

9. Engvall, E. \& Perlmann, P. (1972) J. Immunol. 109, 129135.

10. Schößler, W. \& Ilchmann, D. (1986) DD- $\dot{W} \tilde{P}$ unter WPCO8F 2886478 angemeldet.

11. Köhler, A., Jung, K. \& Pergande, M. (1979) Zbl. Pharm. $118,923-942$.

12. Töpfer, G. (1982) Z. Med. Lab. Diagn. 23, 211-213.

Dr. sc. W. Schößler

Staatliches Institut für

Immunpräparate und Nährmedien

Klement-Gottwald-Allee 317/321

DDR-1120 Berlin 